\title{
Modelado e implementación del tractor John Deere 6125d en la preparación de la tierra para cultivos de arroz
}

\section{Modeling and implementation of the John Deere 6125d tractor in the preparation of land for rice crops}

\author{
Darwin Gregorio Chele Sancan \\ Universidad Internacional del Ecuador, Ecuador \\ Marco Vinicio Noroña Merchán \\ Universidad Internacional del Ecuador, Ecuador
}

Autor para correspondencia: darwinchelesancan@ hotmail.com,mar-1582@ hotmail.com

Fecha de recepción: 16 de Septiembre de 2016 - Fecha de aceptación: 01 de Noviembre de 2016

\section{Resumen}

En el presente artículo se estudia las diferentes aplicaciones que se puede dar a un trator John Deere $6125 \mathrm{D}$ en la preparación de la tierra para el cultivo de arroz. En base a la preparación de suelos se puede utilizar al trator en las diferentes fases de arado, emparejamiento del terreno, fangueo y pases del rototiller (arado rotativo).

De esta manera se podrá sacar provecho a la máquina donde se obtendrá una mejor preparación de terrenos en menor tiempo, obteniendo mayores ganancias. En base a los precios de mercado se realizó la tabla de costos, donde se toma como referencia el valor aproximado, en caso de alquiler de la maquinaria para trabajos similares.

Palabras claves: arado; arroz; cultivo; fangueo; rototiller

\begin{abstract}
In the present article discusses the different applications that you can give to a John Deere 6125D tractor in the land preparation for the cultivation of rice. On basis of soil, preparation can be use the tractor in the different phases of plow, land leveling, puddling, and passes from the rototiller. In this way, you can benefit to the use of the machine and can get a better land preparation in short time, getting better profits. Based on the costs of market is conducted the cost table, where it takes as a reference the approximate value in case of machinery rental for similar jobs.
\end{abstract}

Key words: plow; rice; cultivation; puddling; rototiller 


\section{Introducción}

La producción agrícola es un elemento indispensable para alcanzar el desarrollo económico-social del Ecuador, pues ella debe garantizar la satisfacción de las necesidades alimentarias de la población y asegurar además la creación de materias primas para la elaboración de diferentes artículos industriales.

La misma es inconcebible sin el empleo de la técnica mecanizada (instrumento de gestión de la agricultura), la cual asegura el logro de altas producciones, sobre la base de la humanización del trabajo el cual es agotador cuando se realiza las labores del campo con herramientas manuales, y el aumento de la productividad de este. La eficacia en la mecanización de las operaciones y procesos de producción depende no solo de la perfección técnica de las máquinas, sino también, en grado considerable, de la elección correcta de éstas para la obtención de logros hasta ahora alcanzados como son el aumento de las áreas de producción, reducción de costos y rendimientos considerables, teniendo en cuenta las condiciones concretas del suelo y las demás condiciones tecnológicas de trabajo. "la producción agrícola, como una actividad empresarial, efectúa una inversión, recuperar esa inversión, deducir los otros gastos incurridos y además obtener una utilidad, que le permita que la actividad le sea rentable y ser exitoso, productivo, competitivo y sostenible" (Secretaría de Agricultura y Ganadería, 2003).

La preparación del terreno es de suma importancia ya que esto reducirá las pérdidas de agua y de nutrientes por percolación o infiltración, también para un mejor control de las malezas reduciendo la presencia de plagas y enfermedades para poder obtener un buen manejo, crecimiento y desarrollo del cultivo de arroz el cual se realiza bajo condiciones de terreno seco e inundado, en terreno seco se trabaja con arado y rastra pesada (romplow) y el fangueo donde se reemplaza las ruedas posteriores del tractor por unas estructuras cilíndricas de barras metálicas conocidas como gavies (jaula)que realiza un batido del suelo saturado con agua y parcialmente inundado.

En consecuencia la administración de la maquinaría requiere de conocimientos técnicos y comerciales que los productores deben de manejar y combinar adecuadamente el recurso humano y mecánico además de tecnología, para tomar decisiones acertadas al momento de seleccionar la maquinaria apropiada la cual nos permitirá lograr los objetivos con menor costo sin afectar la integridad física y la salud del operador y del medio ambiente, y contar con una fuerza laboral estable.

\section{Materiales y Métodos}

El continuo crecimiento de la población y por ende de la demanda alimenticia hace necesario buscar nuevas alternativas que ayuden a realizar los trabajos de preparación del suelo para la siembra en cortos periodos de tiempo lo cual es posible con el empleo de máquinas y ciertos implementos que facilitan los trabajos que antes se los realzaba de manera manual. La selección de la máquina e implementos es un factor importante ya que debe de ser el adecuado para el tipo de trabajo a realizar, la máquina tiene que brindar la potencia que se requiere para los implementos utilizados en la preparación de los suelos, que no sea muy pesada, que no requiera 
de operarios altamente capacitados y que además no tiene que ser costosa sino que exista una buena relación costo/beneficio y contar con repuestos y servicio técnico.

\section{Método}

El presente estudio se basa en que el cultivo de arroz, el más extenso en Ecuador del cual el 80\% son productores de hasta 20 hectáreas según el Censo Nacional Agropecuario del 2002, se caracteriza por utilizar tractores de doble tracción y de potencia media (100 a 190 HP) que se comercializan a nivel nacional, mismos que brindan confiabilidad y tecnología, que permite realizar diversas actividades, diseñados para una mayor productividad y desempeño en el campo, de fácil mantenimiento y ergonómico para garantizar un manejo confortable.

"Existe una gran variedad de opciones de labranza......viable y con énfasis en la conservación del suelo, ya que el deterioro progresivo de éste afecta su productividad, la rentabilidad a mediano y largo plazo" (Cortés M., Álvarez M., \& González S., 2009), el sistema de manejo de la producción arrocera depende mucho de factores como el clima, la zona, medios de riego y grado de tecnificación. La preparación del suelo es con arada profunda, rastrada, nivelación y fangueo, la temperatura se considera crítica para el arroz cuando es menor de $20{ }^{\circ} \mathrm{C}$ y mayor de $32{ }^{\circ} \mathrm{C}$, las zonas de mayor producción de arroz son Guayas y Los Rios en invierno en el mes de enero y en verano junio - julio con riego.

\section{Preparación de la Tierra para el Cultivo de Arroz}

La preparación de la tierra para el cultivo es una de las labores agrícolas de mayor importancia debido a que este debe de ser el adecuado para las necesidades de la planta, soporte físico, proveedor de nutrientes durante su crecimiento y desarrollo. En el suelo es donde se desarrollan factores adversos para el cultivo como lo son la malesa, insectos, hongos y bacterias por lo cual el principal objetivo de la preparación de la tierra es destruir estos factores e incorporar materia orgánica y contribuir a mejorar su estructura a fin de que la semilla pueda germinar.

Teniendo "una preparación adecuada del suelo, favorece la reducción de las pérdidas de agua y de nutrientes por lixiviación, percolación o infiltración y también se logra un mejor control de las malezas" (Secretaría de Agricultura y Ganadería, 2003).

Preparación de la tierra en condiciones de suelo seco

Cuando la tierra se cultiva por primera vez o está muy compactada (lo cual impide el normal desarrollo radicular de la planta) se utiliza el arado (figura 1), también se realiza esta labor al final de la cosecha para volcar el suelo y que permanezca así durante la época seca y no es recomendado después de haber nivelado el terreno ya que se puede echar a perder la nivelación causando trastornos en la germinación y durante la cosecha. 


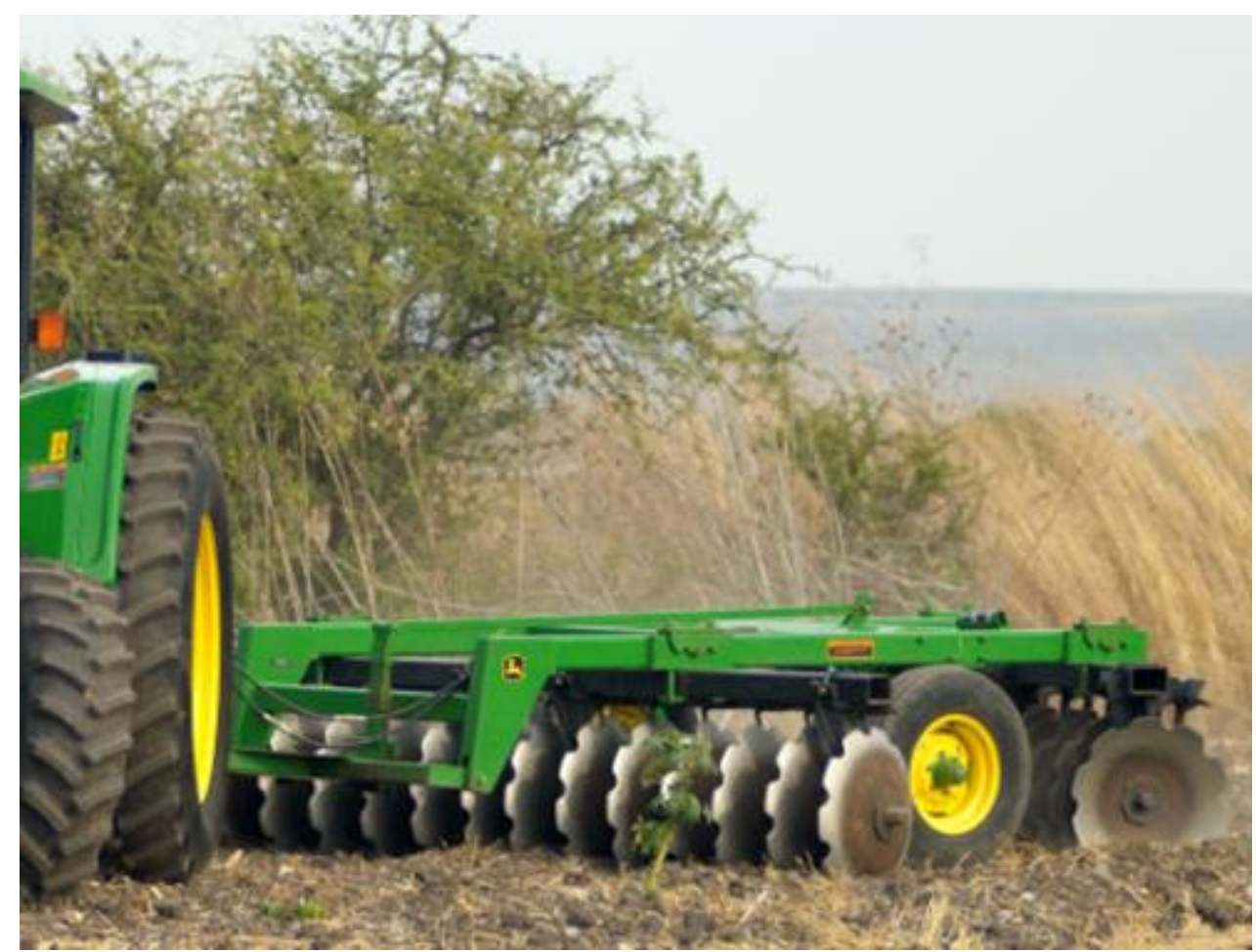

Figura 1. Arado de la tierra

La preparación del suelo para el cultivo de arroz influye de manera directa e indirecta en los rendimientos ya que afecta el uso del agua al momento del riego o fangueo, al uso de fertilizantes y la capacidad de las malezas. Se hace uso de una rastra debido a la versatilidad de este arado, por lo general se efectúa de dos a cuatro pases de rastra pesada para poder lograr una efectiva preparación de tierras para la siembra de arroz. Se tiene como recomendación que se realice el último pase de la rastra antes de la siembra.

Si se tiene un terreno a desnivel lo recomendable es realizar un emparejamiento del terreno mediante un arado que se pueda acoplar al tractor de manera que se consiga una buena distribución de semilla, una profundidad apropiada y tapado de la misma.

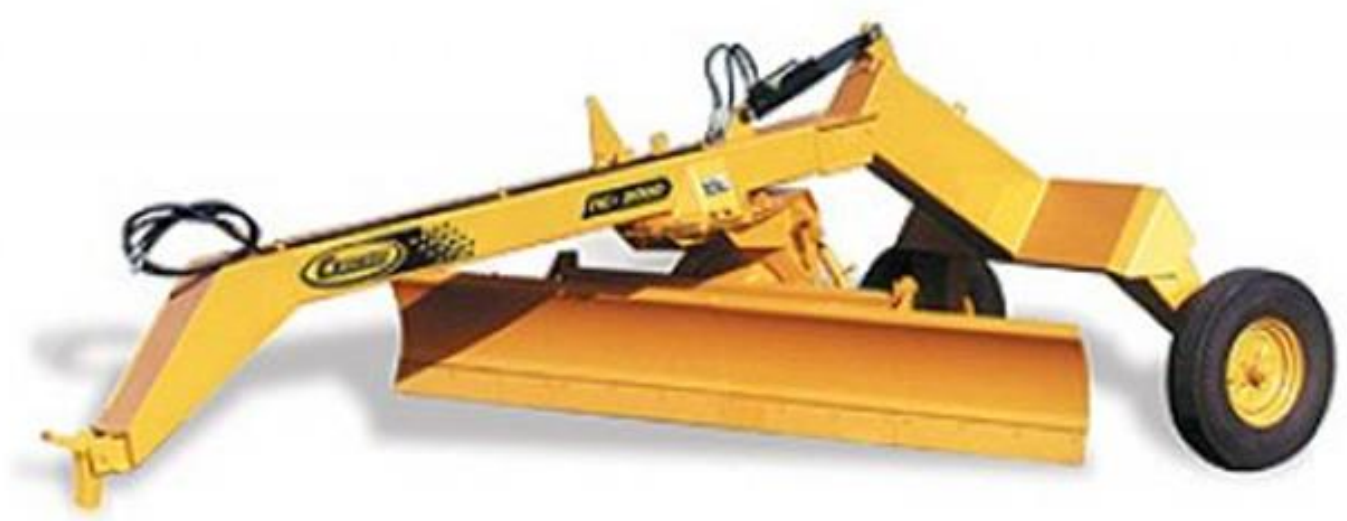

Figura 2. Implemento para la nivelación de terrenos 


\section{Preparación de la tierra en condiciones de fangueo o bajo inundación}

Este tipo de preparación de la tierra se recomienda cuando se tiene los implementos necesarios para el fangueo y un sistema de riego que mantenga una lámina de agua permanente en el cultivo. Este es costoso en comparación con la preparación de la tierra en seco y es recomendable para siembra por trasplante o al voleo con semilla pregerminada. Al realizar la preparación del suelo mediante el fangueo se tiene por objetivo:

a) Mezclar la materia orgánica con el suelo generando nutrientes para el arroz

b) Controlar las malezas

c) Destruir la porosidad y estructura del suelo para evitar la pérdida de agua

d) Convertir el suelo en fango de esta manera se facilita el trasplante o la siembra de semillas germinadas

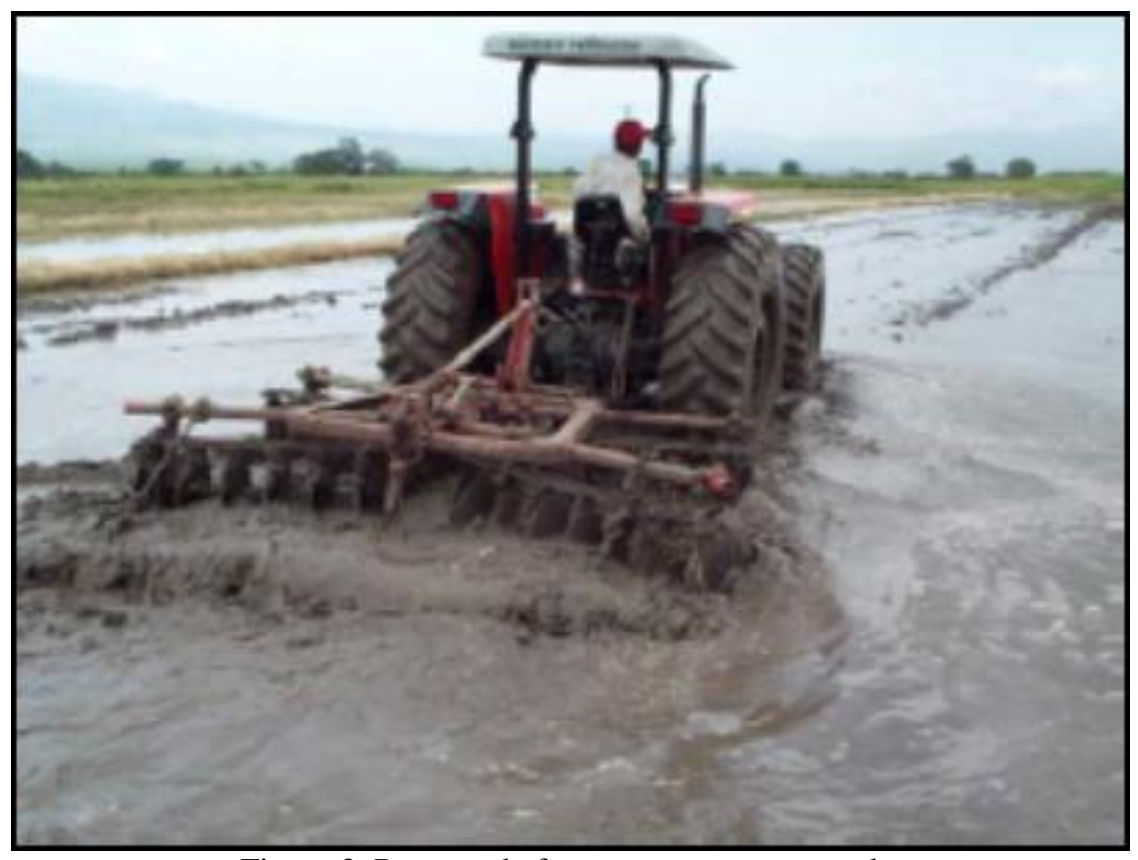

Figura 3. Proceso de fangueo con rastra pesada.

Inundación del terreno y pase del rototiller

Después de limpiar las melgas se utiliza una rastra pesada con uno o dos pases. Una vez se haya realizado el paso de la rastra, las melgas se llenan con una lámina mínima de agua de 20 $\mathrm{cm}$ de profundidad, luego de su llenado se ejecuta el fangueo con una rastra liviana o rototiller teniendo en cuenta las siguientes observaciones:

a) El suelo y el agua deben mezclarse apropiadamente

b) Se debe utilizar las malezas del cultivo anterior convertidos en nutrientes

En caso de no poseer un rototiller3 se recomienda el uso de una rastra liviana. 


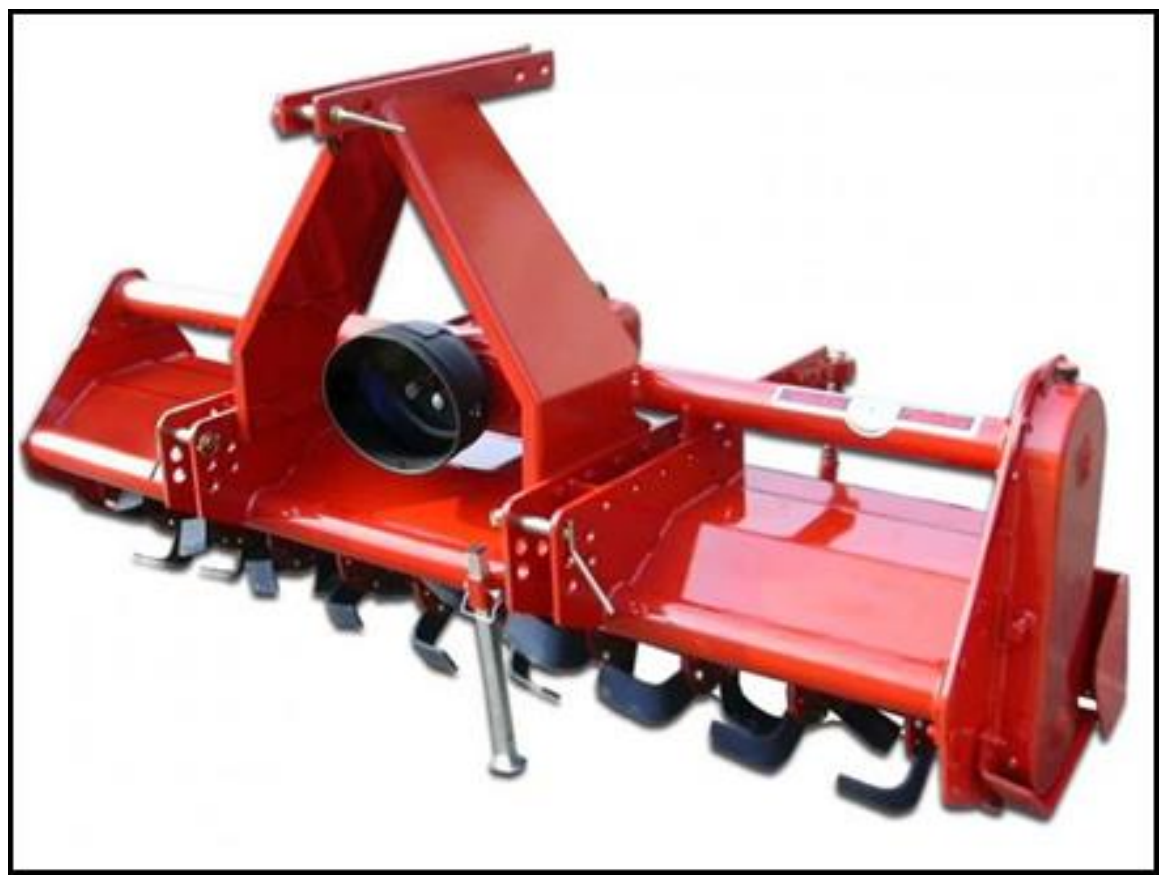

Figura 4. Arado rotativo con enganche de tres puntos.

\section{Características del tractor a utilizar}

El tractor debe ser entre pequeño a mediano, teniendo en cuenta que no se deberá sobrepasar los límites de funcionamiento del tractor durante los trabajos de campo, siendo así el tractor John Deere 6125D que cuenta con las siguientes características:

Tabla 1. Especificaciones técnicas del tractor John Deere 6125D

\begin{tabular}{|c|c|}
\hline \multicolumn{2}{|r|}{ Motor } \\
\hline Serie motor & $6125 \mathrm{D}$ \\
\hline Marca & John Deere \\
\hline Modelo & Power Tech ${ }^{\mathrm{TM}} 4045 \mathrm{H}$ Diesel \\
\hline Potencia & $125 \mathrm{HP}$ \\
\hline Cilindrada & 4,5 litros \\
\hline \multicolumn{2}{|r|}{ Equipamiento } \\
\hline \multicolumn{2}{|c|}{ Barra de tiro } \\
\hline \multicolumn{2}{|c|}{ Anclaje de tres puntos } \\
\hline \multicolumn{2}{|c|}{ Aire acondicionado } \\
\hline \multicolumn{2}{|c|}{ Cabina de seguridad } \\
\hline \multicolumn{2}{|c|}{ Sistema de frenos sellado } \\
\hline \multicolumn{2}{|c|}{ Transmisión } \\
\hline \multicolumn{2}{|c|}{ Doble tracción } \\
\hline \multicolumn{2}{|c|}{ Toma de fuerza (TDF) } \\
\hline Potencia & $105 \mathrm{HP}$ \\
\hline Tipo & Independiente \\
\hline Régimen & $540 / 1000 \mathrm{rpm}$ \\
\hline
\end{tabular}

El significado de $6125 \mathrm{D}$ se representa como sigue: 
Tabla 2. Serie 6125D.

\begin{tabular}{ll}
\hline $\mathbf{6}$ & Serie del tractor (tractor mediano) \\
\hline $\mathbf{1 2 5}$ & Potencia del motor en HP \\
$\mathbf{D}$ & Tipo de tecnología \\
\hline
\end{tabular}

Nota: mientras mayor sea la letra conform el abecedario mayor es la tecnología empleada en el tractor.

Además de estas características se aconseja el uso de ruedas fangueadoras de fácil montaje con la finalidad de evitar que el tractor pierda tracción y se quede atrapado en el terreno. Para mantener la estabilidad y evitar que se produzca un volcamiento del tractor se hace uso de contrapesas ubicadas en la parte delantera, seis y en la parte posterior, cuatro. Las llantas a utilizar son anchas y altas (23.1" x 26" o 23.1" x 30") para aumentar la flotabilidad, para conseguir dicha flotabilidad se retira el agua de las ruedas y se las infla hasta que posean una presión de 5 psi lo que reduce el peso y facilita el movimiento del tractor en el barro.

\section{- Dimensiones}

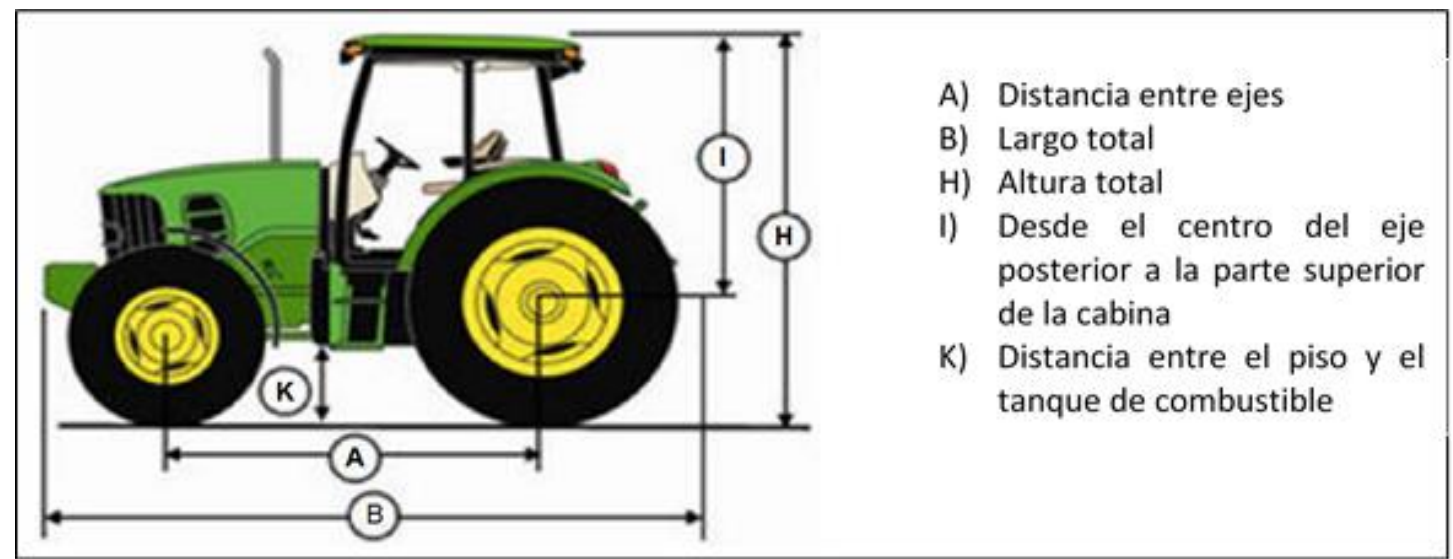

Figura 5. Dimensiones del tractor John Deere 6125D 


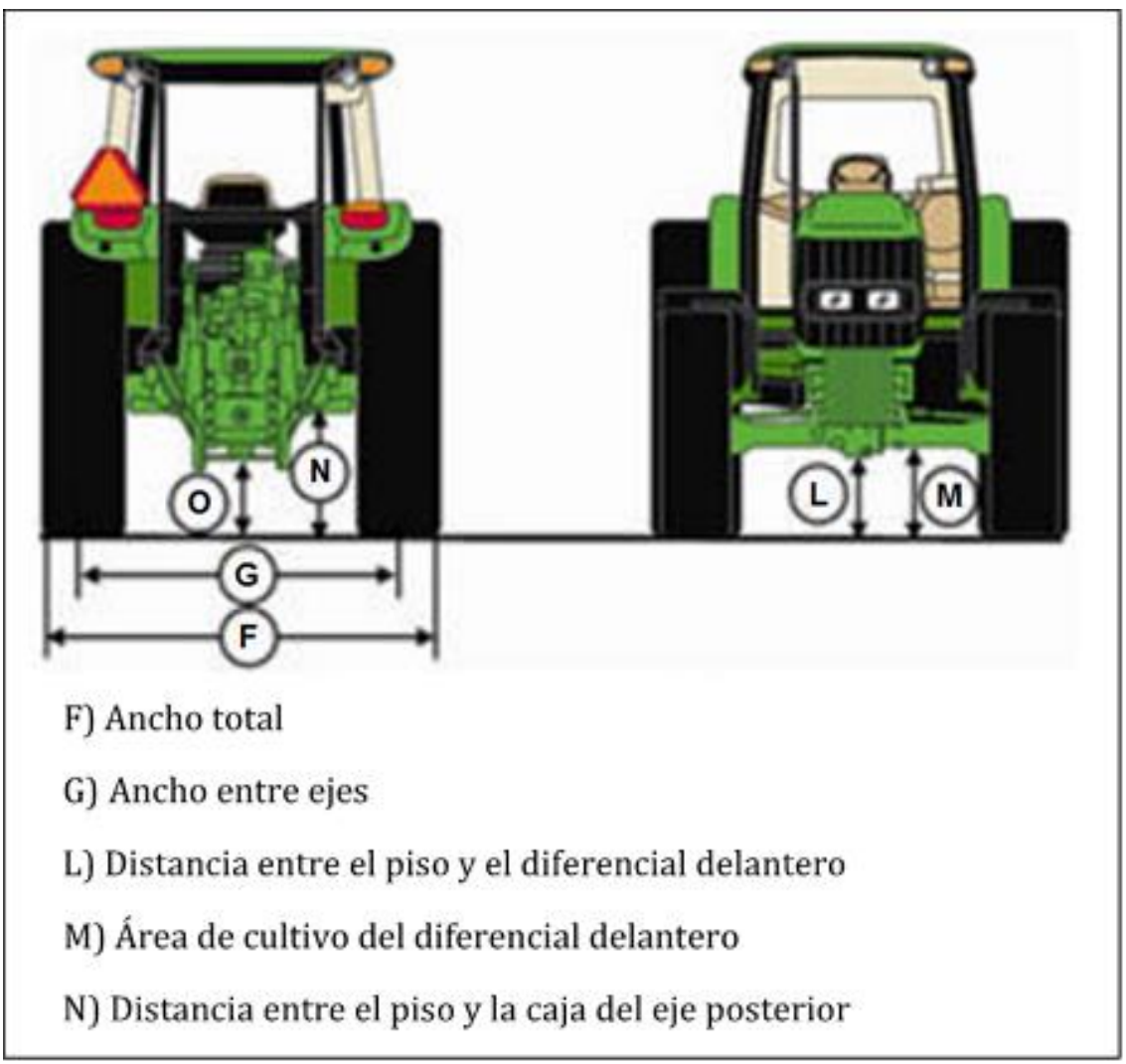

Figura 6. Dimensiones del tractor John Deere 6125D (continuación)

La tabla 3 indica las dimensiones del tractor John Deere 6125D con respecto a las figuras 5 y 6.

Tabla 3. Dimensiones del tractor John Deere 6125D.

\begin{tabular}{lc}
\hline \multicolumn{2}{c}{ Dimensiones (mm) } \\
\hline A & 2316,5 \\
B & 4137,0 \\
H & 2756,0 \\
I & 1976,1 \\
K & 502,9 \\
F & 2451,1 \\
G & 1658,6 \\
L & 1976,1 \\
M & 596,9 \\
$\mathbf{N}$ & 686,0 \\
$\mathbf{O}$ & 515,6 \\
\hline
\end{tabular}

\section{Diseño del tractor y análisis de costos}

El autor ha realizado el modelado del tractor mediante el software AutoCAD de manera de inserción de la imagen del tractor y el repaso o formación del contorno del tractor, dando 
lugar así al modelado en 2D. Posterior se realizó una extrusión del plano 2D transformándolo en 3D, lo que nos ayudaría a darle los detalles necesarios al tractor. En los anexos se podrá observar una presentación de las vistas en 2D y 3D.

\section{Costos de utilización de las máquinas agrícolas}

Mediante la investigación de los costos en el mercado nacional se pudo realizar una tabla destinada a conocer si es rentable para una persona que adquirir un tractor 6125D y su costo por hora en caso que desee alquilar el tractor a otros agricultores de la zona, considerando ciertos factores que puedan variar durante la vida útil de la máquina. Se logra estimar un precio promedio de costos fijos y costos variables presentes en la maquinaria y por último el costo de alquiler por hora como se indicó anteriormente y como se detalla en el anexo.

La vida útil de la maquinaria depende de sus características y forma de realizar su trabajo, esta vida útil se la puede determinar en horas o en años. Dentro de los costos tenemos los costos fijos (amortización, interés, alojamiento, seguros e impuestos) y los costos variables (mano de obra. Combustible y lubricantes) y también se tiene los costos por reparación y mantenimiento, las unidades para representar los costos fijos es dólares/año y los variables dólares/hora o dólares/hectáreas.

- La amortización se puede obtener a partir del valor residual:

$$
\mathrm{Vr}=[\mathrm{C} 1-\mathrm{C} 2(\mathrm{n} 0,5)-\mathrm{C} 3(\mathrm{~h} 0,5)] 2 \mathrm{Va}
$$

Donde:

$\mathrm{Vr}=$ Valor residual

C1, C2 y C3 = Coeficiente cuyo valor depende del tipo de máquina (tabla en el anexo)

$\mathrm{n}=$ Tiempo de la máquina expresada en años

$\mathrm{h}=$ Valor medio del número de horas de uso anual durante esos años

$\mathrm{Va}=$ Valor de adquisición

- La amortización se calcula con la siguiente ecuación:

$$
\mathrm{A}=(\mathrm{Va}-\mathrm{Vr}) / \mathrm{n}
$$

Donde:

A = Amortización

$\mathrm{Va}=$ Valor de adquisición

$\mathrm{Vr}=$ Valor residual

$\mathrm{n}=$ Años de vida de la maquinaria

- La ecuación para calcular el interés que se dejó de percibir con el dinero que se utilizó en la compra de la máquina: 


$$
\mathrm{I}=[(\mathrm{Va}+\mathrm{Vr}) / 2] . \mathrm{i}
$$

Donde:

$\mathrm{I}=$ Costo de interés

$\mathrm{i}=$ Interés dejado de percibir (se puede tomar el interés anual de polizas de acumulación)

- Para el alojamiento la ASABE (American Society of Agricultural and Biological Engineers) propone atribuir una cantidad anual aproximada del $0,75 \%$ del precio de compra de la máquina.

$$
\mathrm{a}=0,0075 . \mathrm{Va}
$$

$\mathrm{a}=$ Alojamiento

$\mathrm{Va}=$ Valor de adquisición

- Los seguros e impuestos depende de la normtiva legal vigente en cada territorio, su valor será un dato perfectamente conocido al hacerse efectivo cada año en uno o varios pagos. Se puede utilizar la siguiente ecuación:

$$
\mathrm{SI}=(\mathrm{Va}+\mathrm{Vr} / 2) * 0,03
$$

Donde:

$\mathrm{SI}=$ Seguros e Impuestos

Nota: se multiplica al valor promedio de la máquina por el 3\% de seguro anual.

- Para el costo de reparación y mantenimiento la ASABE ha elaborado la siguiente ecuación:

$$
\mathrm{RM}=\mathrm{F} 1 . \mathrm{Va}(\mathrm{h} / 100) \mathrm{F} 1
$$

Donde:

$\mathrm{RM}=$ Costo de reparación y mantenimiento

F1 y F2 = Parámetros diferentes para cada tipo de máquinas (tabla en el anexo)

$\mathrm{h}=$ Horas acumuladas de uso

- La ASABE considera que el consumo de combustible medio de los tractores con un motor a diésel se puede calcular con la siguiente ecuación:

$$
\mathrm{Q}=0,2226 . \mathrm{N}
$$

Donde:

$\mathrm{Q}=$ Consumo en $1 / \mathrm{h}$ 
$\mathrm{N}=$ Potencia máxima a la toma de fuerzas en $\mathrm{kW}$

Al resultado se le multiplica por el precio del combustible.

- El costo del lubricante es aproximadamente $15 \%$ del costo del combustible.

- El costo de mano de obra depende del territorio.

Los costos totales se pueden expresar en dólares/año, dólares/hora y dólares/hectáreas empleando las siguientes ecuaciones:

$$
\begin{gathered}
\mathrm{CT}=\mathrm{CF}+\mathrm{CV} \cdot \mathrm{h} \quad[\text { dólares/año] } \\
\mathrm{CT}=(\mathrm{CF} / \mathrm{h})+\mathrm{CV} \quad[\text { dólares } / \mathrm{hora}]
\end{gathered}
$$

Donde:

$\mathrm{CF}=$ Suma de los costos fijos (dólares/año)

$\mathrm{CV}=$ Suma de los costos variables (dólares/hora)

$\mathrm{h}=$ número de horas trabajadas al año

$\mathrm{Se}=$ capacidad de trabajo efectiva (ha/h) de la máquina cuyo costo se está calculando ecuaciones:

Para el cálculo de la capacidad efectiva de trabajo (Se) se utiliza las siguientes

- Capacidad teórica de trabajo

$$
\mathrm{St}=(\mathrm{am} . \mathrm{v}) / 10[\mathrm{ha} / \mathrm{h})]
$$

Donde:

$\mathrm{St}=$ Capacidad teórica de trabajo am = Anchura teórica de la máquina $[\mathrm{m}]$ $\mathrm{v}=$ Velocidad normal de trabajo $[\mathrm{km} / \mathrm{h}]$

- La capacidad de trabajo efectivo se obtiene de la siguiente ecuación:

$\eta \mathrm{e}=\mathrm{Se} / \mathrm{St} \rightarrow \mathrm{Se}=\mathrm{St} . \eta \mathrm{e}$

Reemplazando la capacidad teórica de trabajo queda:

$$
\mathrm{Se}=[(\mathrm{am} . \mathrm{v}) / 10] . \text { Пe }[\mathrm{ha} / \mathrm{h}]
$$

Donde:

$\mathrm{Se}=$ Capacidad de trabajo efectivo 
П $=$ Rendimiento efectivo o de campo (anexo) 
Tabla 4. Cálculo de utilización del tractor

\begin{tabular}{|c|c|c|c|}
\hline \multicolumn{4}{|c|}{ Costo de utilización del tractor john Deere 6125D } \\
\hline Valor de adquisicion (\$) & 71022 & Parámetros para costos de reparación y mant. & \\
\hline Costo de mano de obra $(\$ / \mathrm{h})$ & 1,875 & $\mathrm{~F}_{1}$ & 0,007 \\
\hline Valor residual (\$) & 16642,05 & $\mathrm{~F}_{2}$ & 2 \\
\hline Coeficientes para el calculo de valor residual & & Consumo de combustible $(\mathrm{I} / \mathrm{h})$ & 23,373 \\
\hline$C_{1}$ & 0,976 & Potencia en la toma de fuerza (HP) & 105 \\
\hline $\mathrm{C}_{2}$ & 0,119 & Capacidad de trabajo efectiva (ha/h) & 1,944 \\
\hline $\mathrm{C}_{3}$ & 0,0019 & Capacidad de trabajo teórica (ha/h) & 2,16 \\
\hline Tiempo de la máquina (años) & 15 & Anchura teórica de la máquina (m) & 2,7 \\
\hline Horas promedio de uso anual & 267 & Velocidad normal de trabajo $(\mathrm{km} / \mathrm{h})$ & 8 \\
\hline Interés dejado de persibir (\%) & 5 & Rendimiento efectivo (\%) & 90 \\
\hline Costo del combustible $(\$ / I)$ & 0,2575 & Personal requerido por unidad & 1 \\
\hline \multicolumn{4}{|c|}{ I. Costos fijos } \\
\hline Amortización (\$/año) & 3625,33 & Seguro e Impuestos (\$/año) & 876,64 \\
\hline Costos de interés (\$/año) & 2191,60 & Costo de reparación y mantenimiento & 7974,36 \\
\hline Alojamiento (\$/año) & 532,67 & Total Costos fijo (\$/año) & 15200,60 \\
\hline \multicolumn{4}{|c|}{ II. Costos variables } \\
\hline Combustibles $(\$ / \mathrm{h})$ & 6,02 & & \\
\hline Lubricantes (\$/h) & 0,90 & Total Costo variable $(\$ / \mathrm{h})$ & 8,80 \\
\hline \multicolumn{4}{|c|}{ III. Costos totales } \\
\hline \multirow[t]{2}{*}{ Costo fijo + Costo variable } & & (\$/año) & 17549,22 \\
\hline & & $(\$ / h)$ & 65,73 \\
\hline \multicolumn{4}{|c|}{ V. Precio de alquiler } \\
\hline Costo total $(\$ / \mathrm{h}) *$ margen del $30 \%$ & & & 87,28 \\
\hline
\end{tabular}




\section{Conclusión}

El uso de la maquinaria agrícola facilita las labores de campo, en este caso, en el proceso de siembra de arroz, y en menor tiempo que si se empleara herramientas manuales o con ayuda de animales, motivo por el cual se considera la adquisición de una máquina como una inversión que a la vez se podría poner a disposición (alquiler) de agricultores de la zona.

Cabe destacar que la aplicación de la tecnología ayuda a optimizar el tiempo de trabajo de los tractores además de brindar seguridad y ergonomía al operador.

\section{Bibliografía}

Alvarado Chaves, A. (2004). Maquinaria y mecanización agrícola. San José, C. R.: EUNED.

Cortés M., E., Álvarez M., F., \& González S., H. (2009). La mecanización agrícola: gestión, selección y administración de la maquinaria para las operaciones de campo. CES Medicina Veterinaria y Zootecnia, 4(2), 151-160. Obtenido de http://www.redalyc.org/articulo.oa?id=321428102015

Dávila Cárdenas, R. E. (2005). Administración y planificación de maquinaria agrícola. Caracas: Universidad Central de Venezuela. Obtenido de https://books.google.com.ec

Fountas, S., Sorensen, C. G., Cavalaris, C., Gemtos, T. A., Tsiropoulos, Z., \& Liakos, V. (2015). Farm machinery management information system. Computers and Electronics in Agriculture, 110, 131-138. Obtenido de http://dx.doi.org/10.1016/j.compag.2014.11.011

González Martínez, M. (2011). Mantenimiento, preparación y manejo de tractores (UF 0009). Málaga: IC Editorial. Obtenido de http://www.ebrary.com

Hunt, D., \& Wilson , D. (2016). Farm power \& machinery management: eleventh edition. Estados Unidos de América: Waveland Press. Obtenido de https://books.google.com.ec

Instituto Nacional Autónomo de Investigaciones Agropecuarias. (2007). Manual del cultivo de arroz. En F. Andrade E., R. Celi H., \& J. Hurtado D., Técnicas de cultivo. Guayaquil: INIAP Archivo Historico. Obtenido de https://books.google.com.ec/books?id=IXozAQAAMAAJ\&pg=PA30\&hl=es\&source=gb $\mathrm{s} \_$selected_pages $\&$ cad $=2 \# \mathrm{v}=$ onepage $\& \mathrm{q} \& \mathrm{f}=$ false

John Deere. (2016). 6125D utility tractor features. Obtenido de https://www.deere.com/en_CAF/products/equipment/tractors/utility_tractors/6d_series/61 $25 \mathrm{~d} / 6125 \mathrm{~d}$.page

Osca Lluch, J. M. (2013). Cultivos herbáceos extensivos: cereales. Valencia, ES: Editorial de la Universidad Politécnica de Valencia. Obtenido de http://www.ebrary.com 
Ruiz Cobos, J. M. (2013). Operaciones auxiliares de preparación del terreno, plantación y siembra de cultivos agrícolas (MF0517_1). Málaga, ES: IC Editorial. Obtenido de http://www.ebrary.co

Secretaría de Agricultura y Ganadería. (2003). Manual técnico para el cultivo de arroz (oryza sativa) . Obtenido de https://curlacavunah.files.wordpress.com/2010/04/el-cultivo-delarroz.pdf

Valenzuela A., F., Riquelme S., J., \& Carrasco J., J. (2010). Costos de operación de la maquinaría agrícola. Obtenido de http://biblioteca.inia.cl/biblioteca/resultado.php

Yousif, L. A., Dahab, M. H., \& El Ramlawi, H. (2013). Crop-machinery managementsystem for field operation and farm machinery selection. Journal of Agricultural Biotechnology and Sustainable Development, 5(5), 84-90. Obtenido de http://www.academicjournals.org/JABSD 